\title{
Anterior optic nerve microvascular changes in human glaucomatous optic neuropathy
}

\begin{abstract}
The microvascular changes in the anterior optic nerve in human glaucomatous eyes were examined by selective methylmethacrylate microvascular corrosion castings following cannulation of the central retinal artery and posterior ciliary arteries in $\mathbf{1 1}$ normal eyes and 9 glaucomatous eyes. The resulting castings were examined with scanning electron microscopy. Microvascular changes were found in the anterior optic nerves of all the glaucomatous eyes with visual function loss. These findings include areas of capillary filling defects within the anterior optic nerve and a decreased numbers of feeding arteriolar vessels to the anterior optic nerve. In the prelaminar and laminar regions, the typical capillary patterns are lost and laminar striations are not present. Juxtapapillary choroidal and retinal avascular areas were also identified in two of the glaucomatous eyes. Selective microvascular corrosion casting is an excellent method to examine the threedimensional microvasculature of the anterior optic nerve. Microvascular changes in the anterior optic nerve may play a role in the development of glaucomatous optic neuropathy.
\end{abstract}

Key words Glaucoma, Microcirculation, Optic nerve

The pathogenesis of glaucomatous optic neuropathy has been studied extensively and regional microvascular changes have been cited as a potential contributor. The microvasculature of the anterior optic nerve in glaucoma has been the subject of histological, clinical and experimental investigations. Many studies have suggested that vascular factors can contribute to glaucomatous optic neuropathy. ${ }^{1-6}$

A variety of investigational techniques have been developed to examine the anterior optic nerve microvasculature and blood flow, both in vitro and in vivo. ${ }^{2-11}$ Selective microvascular corrosion casting techniques are an established method to study the three-dimensional vascular patterns of the anterior optic nerve. The microvasculature of the human anterior optic nerve in normal and glaucomatous eyes has been described, using selective cannulation methods and methylmethacrylate. ${ }^{2,7}$ The purpose of the current study was to investigate the microvascular casting changes of the anterior optic nerve in the human glaucomatous eye.

\section{Materials and methods}

Human eye bank eyes, with at least $10 \mathrm{~mm}$ long retrobulbar optic nerves (allowing identification of the central retinal artery and the posterior ciliary arteries), were obtained within $24 \mathrm{~h}$ of death. All procedures followed the tenets of the Declaration of Helsinki.

Eleven normal eyes were obtained from 6 Caucasian subjects ranging from 63 to 83 years of age (mean age $74.9 \pm 7.9$ years) which were $20.5 \pm 8.7 \mathrm{~h}$ (average time after death) postmortem. Nine glaucomatous eyes were obtained from 5 subjects 70 to 88 years of age (mean age $78.4 \pm 7.5$ years) which were $20.9 \pm 6.3$ hours post-mortem. No pre-mortem systemic vascular disorders or diabetes were found in the normal subjects.

Using a previously described method to selectively cannulate the posterior ciliary arteries and central retinal arteries vascular corrosion castings were developed. ${ }^{2,7,12}$ Pulled polyethylene tubing attached to a 21 gauge needle with tapered tip and MicroFil tubes (World Precision Instruments, Sarasota, FL) were used to cannulate the central retinal artery and posterior ciliary arteries. The tubings were ligated in place by 7-0 or 9-0 nylon sutures. Tissue plasminogen activator (Activase, Genetech, South San Francisco, CA) was injected to flush the microvessels. After flushing four times with tissue plasminogen activator, modified Batson's No. 17 methylmethacrylate medium $^{12}$ (Polysciences, Warrington, PA), the viscosity of which is about $11 \mathrm{cPs}$, was manually injected by $1 \mathrm{ml}$ plastic syringe until the plastic material was observed outflowing from the
D.-Y. Zhao

G.A. Cioffi

Discoveries in Sight Devers Eye Institute Legacy Health System Portland Oregon, USA

George A. Cioffi, MD Glaucoma Service Devers Eye Institute 1040 NW 22nd Ave., Suite 200

Portland, OR 97210, USA

Tel: + 1 (503) 4138203

Fax: +1 (503) 2744839

e-mail: GACioffi@

discoveriesinsight.org

Supported by: NIH Grant EY05231 (G.A.C.) and Glaucoma Research Foundation, San Francisco, CA (D.-Y.Z.)

Presented in part at the Annual Meeting of the Association for Research in Vision and Ophthalmology, Fort Lauderdale, Florida, May 1999 


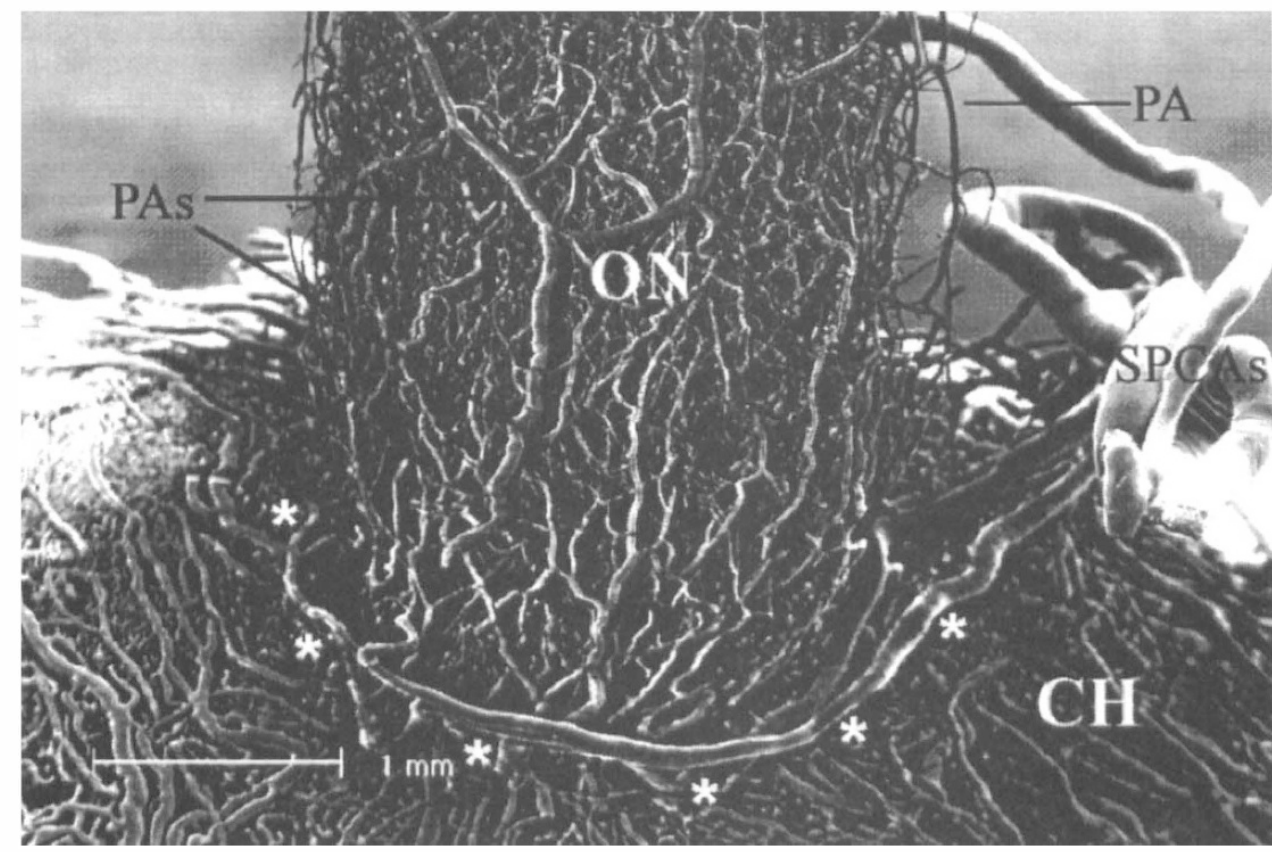

Fig. 1. Methylmethacrylate vascular casting of the right eye of an 83-year-old male. Posterior view shows complete vascular filling of the circle of Zinn-Haller (asterisks) and the anterior optic nerve (ON) as the ON enters the posterior aspect of the eye. Note the multiple 'feeding vessels' branching from the circle of Zinn-Haller and supplying the anterior optic nerve. SPCAs, short posterior ciliary arteries; PAs, pial arteries; $C H$, choroid.

vortex veins and/or central retinal vein. Two hours later, the eyes were immersed in $10 \%$ buffered formalin for $24 \mathrm{~h}$ to allow complete polymerisation. All the surrounding tissue was corroded in $6 \mathrm{M}$ potassium hydroxide $\left(45-50{ }^{\circ} \mathrm{C}\right.$ for $\left.24-48 \mathrm{~h}\right)$, and the vascular castings were rinsed with running water and then airdried completely at room temperature.

Under a binocular dissecting microscope, each casting was oriented and the blood supply to the anterior optic nerve identified. Microdissection was carefully performed to remove the extravasation and to show the detail of the anterior optic nerve. Microdissected castings were mounted on aluminium stubs, sputter-coated with gold-palladium (30 $\mathrm{nm}$ ), and examined under a working accelerated voltage of $2 \mathrm{kV}$, in a scanning electron microscope of total accelerated voltage $30 \mathrm{kV}$ (Philips XL30, The Netherlands). Imaging photographs were taken.

\section{Results}

\section{Normal microvasculature of the anterior optic nerve}

Among the 11 normal eyes, the anatomy of the microvasculature was similar to our previous studies with normal human eyes. ${ }^{7}$ The microvasculature of the normal human anterior optic nerve clearly demonstrated complete microvascular filling of the anterior optic nerve

Table 1. The number of feeding arterioles from short posterior ciliary arteries and the circle of Zinn-Haller to the anterior optic nerve in normal eyes $(n=5)$

\begin{tabular}{lcc}
\hline & \multicolumn{2}{c}{ No. of vessels } \\
\cline { 2 - 3 } & Superior quadrant & Inferior quadrant \\
\hline Normal eyes & $9.6 \pm 1.5$ & $10.8 \pm 1.9$ \\
\hline
\end{tabular}

Values are the mean \pm SD. and the circle of Zinn-Haller (Fig. 1). The anterior optic nerve can be divided into four anatomical regions: the superficial nerve fibre layer, the prelaminar region, the lamina cribrosa and the retrolaminar region (Fig. 2). The superficial nerve fibre layer is supplied from branches of the central retinal arteries. Branches either from the circle of Zinn-Haller or from the short posterior ciliary arteries provide the primary blood supply to the prelaminar and laminar regions. In the prelaminar and lamina cribrosa regions, most of the capillaries run parallel to the posterior sclera. The capillaries in these regions are within the connective tissue plates and therefore have a striated appearance. In the normal casting specimens, we found the number of feeding arteriolar vessels supplying the anterior optic nerve (prelaminar and laminar regions) to be $9.6 \pm 1.5$ (mean \pm SD) and $10.8 \pm 1.9$ in the superior and inferior quadrants of the anterior optic nerve, respectively (Table 1 ). The retrolaminar region is supplied by branches of the short posterior ciliary arteries and pial arteries, as well as occasional branches of the central retinal artery.

\section{Anterior optic nerve microvascular changes in glaucomatous optic neuropathy}

Table 2 illustrates the cannulation sites and pre-mortem clinical data for the 9 glaucomatous eyes. The age of the patients ranged from 70 to 88 years (mean ages $78 \pm 6.5$ ) with a history of glaucoma of 7-19 years. Seven of 9 eyes had pre-mortem visual function changes consistent with glaucoma. Both the medial and lateral posterior ciliary arteries and central retinal artery were cannulated in 8 of 9 eyes. The central retinal artery could not be identified 
Table 2. Cannulation sites and clinical data of the glaucomatous eyes

\begin{tabular}{cccccccc}
\hline $\begin{array}{c}\text { Age } \\
\text { (years) }\end{array}$ & Sex & Diagnosis & Eye & Cannulation & $\begin{array}{c}\text { Max. IOP } \\
\text { (mmHg) }\end{array}$ & C/D ratio & Visual fields \\
\hline 70 & M & POAG & OD & PCAs & 40 & 0.6 & Inferior arcuate \\
70 & M & POAG & OS & CRA + PCAs & 40 & 0.7 & Inferior arcuate \\
74 & M & POAG & OD & CRA + PCAs & 38 & 0.9 & Severe visual loss \\
74 & M & POAG & OS & CRA + PCAs & 23 & 0.5 & WNL \\
83 & F & POAG & OD & CRA + PCAs & 23 & 0.6 & WNL \\
83 & F & POAG & OS & CRA + PCAs & 36 & 0.7 & Scotoma \\
81 & F & POAG & OD & CRA + PCAs & 30 & $>0.95$ & Blind \\
81 & F & POAG & OS & CRA + PCAs & 30 & 0.9 & Constriction \\
88 & M & POAG & OS & CRA + PCAs & 36 & 0.7 & Superior and inferior
\end{tabular}

POAG, primary open-angle glaucoma; OD, rigid eye; OS, left eye; PCAs, posterior ciliary arteries; CRA, central retinal artery; C/D ratio, cup/disc ratio; WNL, within normal limitation.

in 1 eye. All the eyes with visual function loss demonstrated microvascular changes. There were three principal findings in the glaucomatous eyes:

1. The anterior optic nerves had areas of capillary loss and fewer feeding arteriolar vessels (range 3-6) in all eyes with visual function defects (Fig. 3).

2. In the prelaminar and laminar regions, the typical capillary patterns or striations were lost (Fig. 2).

Capillary dropout mainly occurred within the prelaminar and laminar regions of the anterior optic nerve.

3. Juxtapapillary choroidal and retinal avascular areas were found in 2 of the glaucomatous eyes.
Anterior optic nerve microvascular changes have been demonstrated in primary open-angle glaucoma in a variety of studies. ${ }^{2-5,13,14}$

Previous studies have suggested an in vivo reduction of blood flow in the juxtapapillary retina and neuroretinal rim area of the glaucomatous optic nerve head, and a reduction in the neuroretinal rim blood flow that is proportional to the increase in the cup/disc ratio. ${ }^{3}$ In another study using retinal flowmetry, $63 \%$ of glaucoma patients had avascular areas in the lamina cribrosa. ${ }^{4}$ This finding suggests that glaucoma patients tend to have smaller blood volume, flow and velocity in the lamina cribrosa. Hayreh ${ }^{14}$ found a reduction in fluorescence of the optic disc in a large number of glaucoma patients with significant changes at the optic disc and visual field defects by using fluorescence angiography. Clinical observations also support the theory that arterial insufficiency and decreased blood flow in the anterior portions of the optic nerve are potential factors in the development of visual field loss in glaucoma. ${ }^{15}$

\section{Discussion}

Selective vascular casting in normal and glaucomatous anterior optic nerve provides three-dimensional models of the microvasculature of the anterior optic nerve.

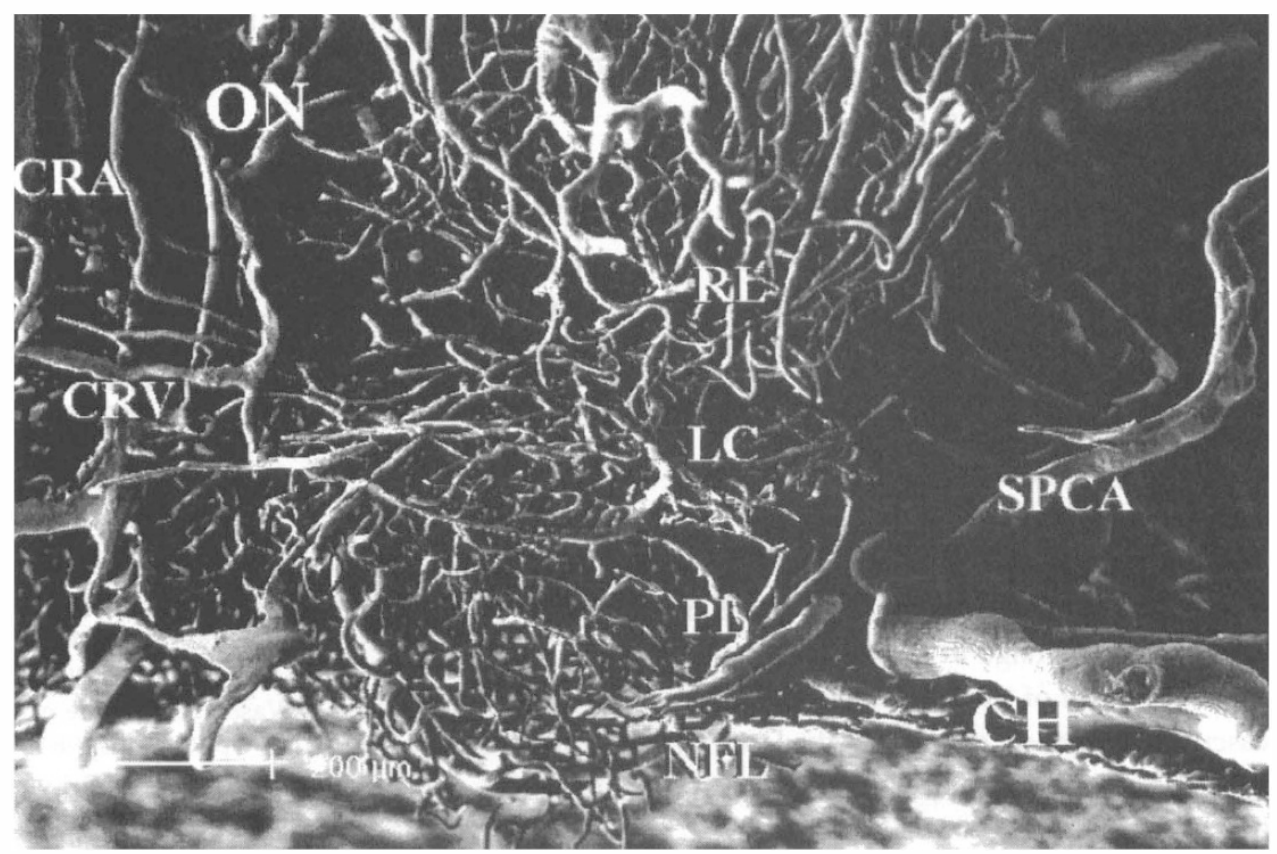

Fig. 2. Posterior view of a microdissected vascular casting of the anterior optic nerve from a 76-year-old female, demonstrating the anatomical regions and blood vessels of the hemisection of optic nerve. NFL, nerve fibre layer; $P L$, prelaminar region; $L C$, laminar cribrosa; $R L$, retrolaminar region; ON, optic nerve; $C R A$, central retinal artery; $C R V$, central retinal vein; SPCAs, short posterior ciliary artery; $C H$, choroid. 


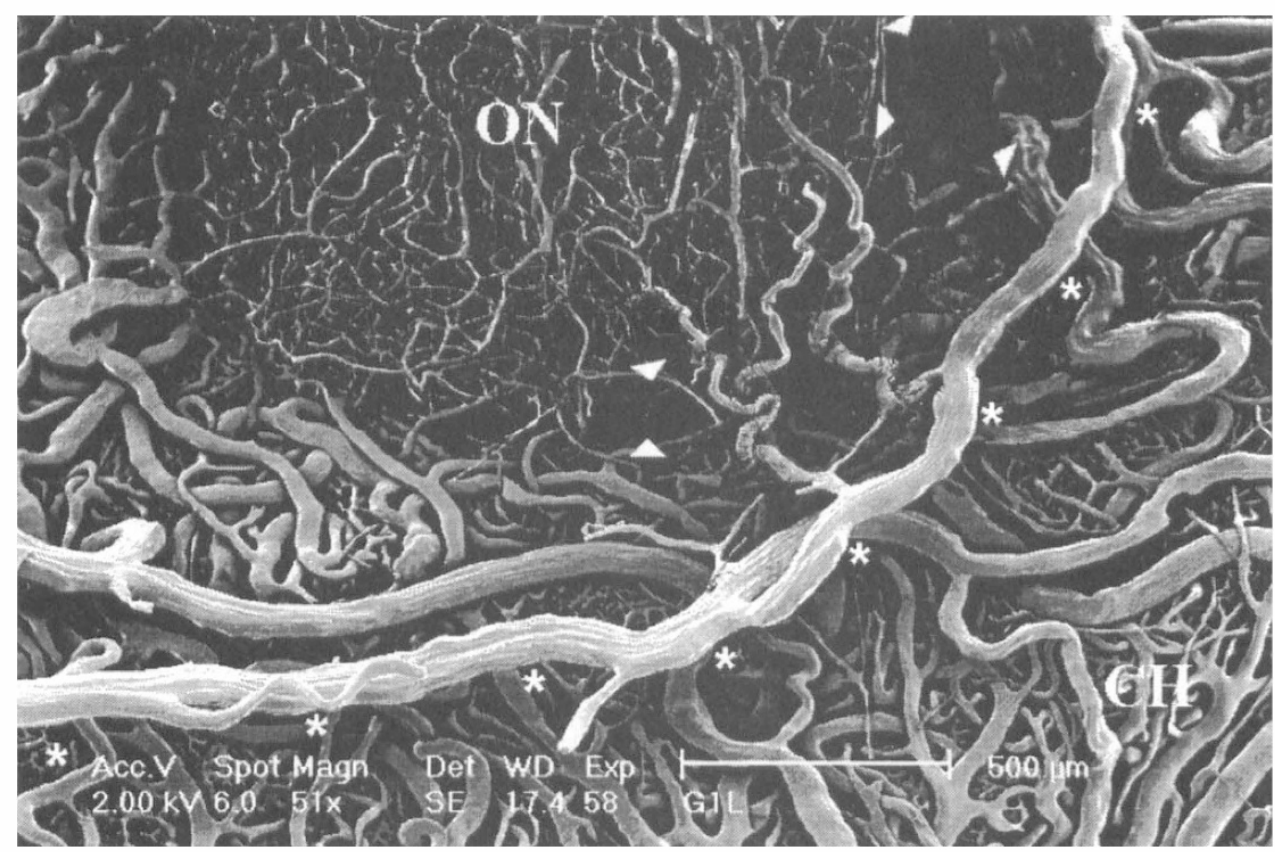

FIg. 3. Vascular casting of the anterior optic nerve from a 70-year-old male, with a glaucoma history of 19 years in the left eye. This view shows an irregular area of a capillary filling defect (within the arrowheads) from the superior temporal to superior nasal aspect. Also note the loss of feeding vessels from the circle of Zinn-Haller in the same region. Asterisks, the circle of Zinn-Haller; $\mathrm{CH}$, choroid; ON, optic nerve.

In experimental glaucoma, François and Neetens ${ }^{15}$ reported that there was significant reduction in the filling of the capillaries of the retina and choroid, and in the optic nerve the reduction was greater on the temporal side. This suggests that an alteration in the blood flow associated with increased intraocular pressure may be a pathological change in the optic nerve. ${ }^{5,16}$ In addition, Hayreh and colleagues ${ }^{17}$ found that $70 \%$ of the choriocapillaris and $67 \%$ of the temporal peripapillary choroid showed moderate to severe atrophy.

Quigley and his co-workers ${ }^{18}$ suggested that the loss of nerve fibres within the anterior optic nerve leads to capillary loss and that a constant relationship between the tissue and capillary ratio was maintained after complete transection of optic nerve. This was also seen in human glaucomatous eyes. ${ }^{13}$

In the current study, microvascular changes were found in the anterior optic nerve and juxtapapillary choroid and retina in the glaucomatous eyes. This suggested that ischaemic changes may be associated with the development of glaucomatous optic neuropathy. However, these post-mortem anatomical observations can not determine whether vascular changes lead to the neuropathy or the neuropathy leads to the vascular changes. It is important to provide sufficient blood flow to the anterior optic nerve so that normal visual function can be maintained. As the damage in glaucoma is believed to occur at the level of the lamina cribrosa, ${ }^{19,20}$ the finding of vascular anatomical changes at the laminar level further supports the ischaemia-induced neuropathy theory. Capillaries may be altered or collapsed by compressive changes of the laminar cribrosa. The loss of typical striated patterns within the anterior optic nerve may be associated with the changes in the laminar plates during the development of glaucomatous optic nerve neuropathy. However, a causal relationship remains to be proven.

To our knowledge, the present study is the first to employ vascular casting techniques in human glaucomatous eyes to illustrate the three-dimensional microvascular changes associated with glaucomatous optic neuropathy. Microvascular changes were found in the anterior optic nerve and also the juxtapapillary choroid and retina of the glaucomatous eyes. It is possible that haemodynamic changes resulting from anatomical microvascular changes of the anterior optic nerve play an important role in the pathogenesis of glaucomatous optic neuropathy.

\section{References}

1. Van Buskirk EM, Cioffi GA. Glaucomatous optic neuropathy. Am J Ophthalmol 1992;113:447-542.

2. Cioffi GA, Zhao DY, Wang L, Van Buskirk EM, Bacon DR. Microvasculature of the human glaucomatous anterior optic nerve. Invest Ophthalmol Vis Sci (ARVO Abstracts) 1999;40:S275.

3. Michelson G, Langhans MJ, Groh MJM. Perfusion of the juxtapapillary retina and neuroretinal rim area in primary open-angle glaucoma. J Glaucoma 1996;5:91-8.

4. Nicolela MT, Hnik P, Drance SM. Scanning laser Doppler flowmeter study of retinal and optic disk flow in glaucomatous patients. Am J Ophthalmol 1996;122:775-83.

5. François J, Neetens A. Vascularity of the eye and the optic nerve in glaucoma. Arch Ophthalmol 1964;71:219-25.

6. Wang L, Cioffi GA, Van Buskirk EM. The vascular pattern of the optic nerve and its potential relevance in glaucoma. Curr Opin Ophthalmol 1998;9:24-9.

7. Onda E, Cioffi GA, Bacon DR, Van Buskirk EM. Microvasculature of the human optic nerve. Am J Ophthalmol 1995;120:92-102. 
8. Hayreh SS. Von Sallman lecture 1994. The optic nerve head circulation in health and disease. Exp Eye Res 1995;61:259-72

9. Zhao Y, Li F. Microangioarchitecture of the optic papilla. Jpn J Ophthalmol 1987;31:147-59.

10. Olver JM, Spalton DJ, McCartney ACE. Quantitative morphology of human retrolaminar optic nerve vasculature. Invest Ophthalmol Vis Sci 1994;35:3858-66.

11. Lieberman MF, Maumenee AE, Green WR. Histologic study of the vasculature of the anterior optic nerve.

Am J Ophthalmol 1976;82:405-23.

12. Fahrenbach WH, Bacon DR, Morrison JC, Van Buskirk EM Controlled vascular corrosion casting of the rabbit eye. J Electron Microsc Tech 1988;10:15-26.

13. Quigley HA, Hohman RM, Addicks EM, Green WR. Blood vessels of the glaucomatous optic disc in experimental primate and human eyes. Invest Ophthalmol Vis Sci 1984;25:918-31.

14. Hayreh SS. Blood supply of the optic nerve head and its role in optic nerve atrophy, glaucoma, and oedema of the optic disc. Br J Ophthalmol 1969;53:721-48.
15. Harrington DO. The pathogenesis of the glaucoma field: clinical evidence that circulatory insufficiency in the optic nerve is the primary cause of visual field loss in glaucoma. Am J Ophthalmol 1959;47:177-85.

16. Kalvin NH, Hamasaki DI, Gass JDM. Experimental glaucoma in monkeys. II. Studies of intraocular vascularity during glaucoma. Arch Ophthalmol 1966;76:94-103.

17. Hayreh SS, Pe'er J, Zimmerman MB. Morphologic changes in chronic high-pressure experimental glaucoma in rhesus monkeys. J Glaucoma 1999;8:56-71.

18. Quigley HA, Hohman RM, Addicks EM. Quantitative study of optic nerve head capillaries in experimental optic disk pallor. Am J Ophthalmol 1982;93:689-99.

19. Anderson DR, Hendrickson A. Effect of intraocular pressure on rapid axoplasmic transport in monkey optic nerve. Invest Ophthalmol Vis Sci 1974;13:771-83.

20. Quigley HA, Addicks EM, Green WR, Maumenee AE. Optic nerve damage in human glaucoma. II. The site of injury and susceptibility to damage. Arch Ophthalmol 1981;99:635-49. 